1 Department of Primary Care \& Public Health, Imperial College London.

Twitter @Azeem_Majeed

Cite this as: BMJ 2022;376:0184 http://dx.doi.org/10.1136/bmj.o184 Published: 21 January 2022

\title{
Reducing the covid-19 isolation period in England: a policy change that needs careful evaluation
}

\section{Michael Soljak, ${ }^{1}$ Azeem Majeed}

How long people with covid-19 should self-isolate depends on the period for which they remain infectious. On 4 January, the US Centers for Disease Control (CDC) updated covid-19 isolation and quarantine recommendations with shorter isolation (for asymptomatic and mildly ill people) and quarantine periods of five days to focus on the period when a person is most infectious, followed by continued masking for an additional five days. ${ }^{1}$ This policy was based on a modelling study from the United Kingdom by Bays et al which showed that after the fifth day after a positive test, an estimated $31 \%$ of people remained infectious. ${ }^{2}$ All the authors of this modelling study, which was published as a pre-print on 24 December 2021, work for UK Health Security Agency (UK HSA).

On 22 December 2021, the UK HSA reduced self-isolation for covid-19 cases in England from 10 to seven days following negative lateral flow tests on days six to seven. The UK HSA stated that a seven day isolation period alongside two negative lateral flow tests had nearly the same protective effect as a 10 day isolation period without testing for people with covid-19.

On 1 January, the UK HSA published a blog on using lateral flow tests to reduce the self-isolation period. ${ }^{3}$ The blog provides background to explain the reasons for the difference between the policies. It also states that after 10 days self-isolation, $5 \%$ of people will still be infectious; and that modelling suggests that ending self-isolation after seven days and two negative lateral flow tests results in a similar level of protection.

The two negative test results are essential in safely supporting the end of self-isolation. Without testing, modelling suggests that $16 \%$ of people would still be infectious after day seven. ${ }^{3}$ On 13 January, Sajid Javid, the Secretary of State for Health and Social Care, stated that from 17 January people will be able leave isolation from the start of day six after two negative lateral flow tests on days five and six.

Both the US CDC and the UK HSA have based their length of isolation policy mainly on a single modelling study by Bays et al. ${ }^{2}$ The data on which the modelling was based is therefore very important. Bays et al provide a single reference for "infectious period distribution," a UKHSA modelling paper by Birrell et al published on 31 May 2021. ${ }^{4}$ Hence, it did not contain any information about the omicron variant of SARS-CoV-2. It gives as a data source: "the Wuhan outbreak additionally provides information on epidemiological parameters: the duration of infectiousness, the mean time from infection to symptom onset; the probability of dying given infection and the mean time from symptoms onset to death."

The Wuhan report by Li et al was published in the New England Journal of Medicine on 26 March 2020. ${ }^{5}$ It does not contain any empirical information on the time for which cases were infectious. It only estimates the mean serial interval (MSI), based on six cases only, which represents the average time between the time of symptom onset of a primary case and that of a secondary case. ${ }^{6}$ The MSI is widely used in infectious disease surveillance and control because it allows investigators to identify epidemiological links between cases and to diagnose new cases that have such epidemiological links with

laboratory-confirmed cases. The MSI in Li et al is 7.5 \pm 3.4 days ( $95 \%$ CI, 5.3 to 19). There is no information specifically about infectious periods.

Policies in both the UK and US are based on limited data and only on the wild-type SARS-CoV-2 variant. Ideally, there should be population-based studies which include daily monitoring of culturable omicron variant viral shedding (or even better actual transmission, which should be available from large databases) and PCR and lateral flow testing. A 2020 (so pre-Delta) rapid scoping review and analysis from Ireland of available evidence for serial testing asymptomatic and symptomatic cases showed substantial variation in the estimates, and how the infectious period was inferred. ${ }^{7}$ The only currently available study of the omicron variant is a small Japanese preprint which showed that people with the omicron variant of covid-19 shed the virus for longer after symptoms emerge. ${ }^{8}$ The number and percentage of omicron variant virus isolation positive samples as $7 / 17(41.2 \%)$ after three to six days and $2 / 18(11.1 \%)$ at seven to nine days. ${ }^{9}$

The change in isolation policy for people with covid-19 in England is a pragmatic step that will allow people to return to productive work, education, and social activities more quickly. People may also be more likely to comply with a shorter isolation period. But the changes should have been based on careful monitoring and review based on new data on the omicron variant, not on data on the wild type of SARS-CoV-2. We therefore need careful evaluation of the new shorter isolation period to ensure that people are following the guidance on self-testing and symptoms, and not ending their isolation period too early, and thereby putting others at risk of infection from covid-19. Finally, non-pharmaceutical interventions should be considered-rapidly-by the National Institute for Health and Care Excellence, which would ensure scientific rigour and cost-effectiveness. 


\section{OPINION}

Provenance and peer review: not commissioned, not peer reviewed.

AM is supported by the NIHR Applied Research Collaboration NW London. The views expressed are those of the author and not necessarily those of the NHS, the NIHR or the Department of Health and Social Care.

$1 \quad$ US Centers for Disease Control. What We Know About Quarantine and Isolation: Why CDC Shortened Isolation and Quarantine for the General Population: US Centers for Disease Control, 2022 https://www.cdc.gov/coronavirus/2019-ncov/if-you-are-sick/quarantine-isolation-background.html.

2 Bays D, Whiteley T, Pindar M, etal. Mitigating isolation: The use of rapid antigen testing to reduce the impact of self-isolation periods.medRxiv 2021:2021.12.23.21268326.

doi: 10.1101/2021.12.23.21268326

3 UK Health Security Agency. Using lateral flow tests to reduce the self-isolation period: UK Health Security Agency, 2022. https://ukhsa.blog.gov.uk/2022/01/01/using-lateral-flow-tests-to-reducethe-self-isolation-period/

4 Birrell P, BlakeJ, van Leeuwen E, et al. Real-time nowcasting and forecasting of COVID-19 dynamics in England: the first wave. Philosophical Transactions of the Royal Society B: Biological Sciences. 2021;376(1829). doi: 10.1098/rstb.2020.0279

5 Li Q, Guan X, Wu P, etal. Early Transmission Dynamics in Wuhan, China, of Novel Coronavirus-Infected Pneumonia. N Engl/ Med 2020;382:1199-207.

doi: 10.1056/NEJMoa2001316. pmid: 31995857

6 Vink MA, Bootsma MCJ, Wallinga J. Serial intervals of respiratory infectious diseases: a systematic review and analysis. AmJ Epidemiol2014;180:865-75. doi: 10.1093/aje/kwu209. pmid: 25294601

7 Byrne AW, McEvoy D, Collins AB, etal. Inferred duration of infectious period of SARS-CoV-2: rapid scoping review and analysis of available evidence for asymptomatic and symptomatic COVID-19 cases. BMJ Open 2020;10:e039856.

doi: 10.1136/bmjopen-2020-039856."https://pubmed.ncbi.nlm.nih.gov/32759252" pmid: 32759252

8 Torjesen I. Covid-19: Peak of viral shedding is later with omicron variant, lapanese data suggest. BMJ 2022;376:089.

doi: 10.1136/bmj.089."https://pubmed.ncbi.nlm.nih.gov/35027360" pmid: 35027360

9 National Institute of Infectious Diseases Disease Control and Prevention Center. Active epidemiological investigation on SARS-CoV-2 infection caused by Omicron variant (Pango lineage B.1.1.529) in Japan: preliminary report on infectious period: National Institute of Infectious Diseases Disease Control and Prevention Center, National Center for Global Health and Medicine, 2022. https://www.niid.go.jp/niid/en/2019-ncov-e/10884-covid19-66-en.htmL 